\title{
CJEM Debate Series: \#HallwayMedicine - Our responsibility to assess patients is not limited to those in beds; emergency physicians must assess patients in the hallway and the waiting room when traditional bed spaces are unavailable
}

\author{
Grant Innes, MD*; Merril Pauls, MD, MHSc ${ }^{\dagger}$; Samuel G. Campbell, MB BCh; Paul Atkinson, MB, MA ${ }^{\ddagger}$
}

INTRODUCTION

\section{Sam Campbell (@samcampcaranx) and Paul Atkinson (@eccucourse)}

This series of editorials provides CFEM readers with an opportunity to hear differing perspectives on topics pertinent to the practice of emergency medicine. The debaters have been allocated opposing arguments on topics where there is some controversy or perhaps scientific equipoise.

We continue with the topic of hallway and waiting room medicine. As the hospital crowding crisis continues, the strident calls from the emergency department (ED) to get non-emergency patients out of ED beds have fallen on deaf ears. As a result, we face a difficult choice: If the patient can't get to a doctor, should the doctor go to the patient? Is it "unethical" to provide formal medical care in a public space, or perhaps to withhold such care, no matter how incomplete? Is hallway medicine the thin end of the wedge? When the waiting rooms become too congested with patients whom we have already seen, will we be going out into the street, shopping malls, and beyond to provide care? Or perhaps we have become too comfortable with the buffer zone of the waiting room, preferring patients to bear the risk of waiting rather than clinicians risking less than optimal conditions for assessment and treatment. "Duct tape" solutions like hallway medicine are typically Canadian - but don't they hide problems? Why should anyone attempt to fix inpatient congestion in the ED when the emergency physicians have solved the problem by managing without the beds that are blocked?

Grant Innes, former Head of Emergency Medicine at the University of Calgary, argues that it is time to move beyond the constraints of the inner sanctum of the ED and provide treatment wherever we can, with Merril Pauls, emergency physician and ethicist, responding that lowering standards of care and taking shortcuts in the short term will not help us reach long-term solutions for our crowding issues.

Readers can follow the debate on Twitter and vote for either perspective, by going to @CFEMonline or by searching \#CFEMdebate.

\section{For: Grant Innes (@GrantInnesEM)}

"No patient left bebind: How waiting room care improves safety and operational efficiency."

While reviewing a local ED, our group walked along a hallway where a dozen emergency medical services (EMS) crews were parked with their patients, then through a packed waiting room, seeing several patients

From the *Department of Emergency Medicine, University of Calgary, Calgary, AB; †Department of Emergency Medicine, Max Rady College of Medicine, University of Manitoba, Winnipeg, MB; and the ‡Department of Emergency Medicine, Dalhousie University, Halifax, NS.

Correspondence to: Dr. Sam Campbell, Department of Emergency Medicine, Dalhousie University, 1796 Summer St., Halifax, NS B3H 3A7; Email: Samuel.campbell@nshealth.ca

(C) Canadian Association of Emergency Physicians 
in distress. We proceeded inside triage, where I overheard one physician say to another, "I'm going home early. There are no patients to see." How absurd is that?

During a 3-minute assessment, triage nurses cannot determine whether back pain is a dissection, beadache is a subarachnoid, or extremity pain is necrotizing fasciitis. Consequently, waiting rooms are dangerous places, an ongoing source of newspaper headlines, inquests, and judicial inquiries. I have dealt with the fallout of waiting room disasters many times as a department head and a family member. Too often the hospital's response is, "We were full. There's nothing we could do."

Last year, at a Christmas party, a friend asked me to see a woman who had collapsed. Bystanders had propped her in a sitting position, diaphoretic, and confused. I laid her down. Within minutes, she was alert and described a vagal prodrome with previous similar episodes. I asked a few more questions and told her about blood pressure and brain perfusion. We agreed she was safe to remain at the party.

You are an emergency physician. You could assess a patient at a party, a track meet, or in the aisle of a 747 . So why would you refuse to see someone in an ED hallway, spitting distance from stretchers, nurses and all the backup you need? We should see patients wherever we can. It's the right thing to do, it's efficient, and it demonstrates to our patients that we are doing everything we can to help them. There is paradoxical speed and simplicity to seeing patients in waiting rooms. They don't have to be supine under a sheet with an IV, a monitor, oxygen, and a full nursing history before we can assess them. If I see a waiting room patient, I can initiate investigations quickly or discharge them if they don't need any. I can reassure someone with a soft abdomen and distinguish a benign headache from a bad one. I can reduce a pulled elbow or a write a Ventolin prescription. I can clear a C-spine, remove a spine board, discharge the motor vehicle accident victim from their EMS stretcher hours before it might otherwise happen, and I can identify patients who don't need a stretcher. Most important, I can spot time bombs. If I hear a scary chest pain story or feel a pulsatile mass, I can get the patient to a resuscitation room; and if the waiting room exam is insufficient, I can reassess the patient in a stretcher after his or her labs are back.

Some suggest that going into a waiting room will get you sued, but this makes no sense. A lawsuit requires a patient who is angry with his or her doctor and an adverse outcome caused by care delays or inappropriate treatment. Of the 10,000+ patients I have seen in waiting rooms during the last 20 years, almost all have expressed gratitude, none have sued me, and none have said they would rather wait longer until a more dignified exam space became available. Sick patients can have adverse outcomes, regardless where they are initially assessed, but if this happens despite an expedited waiting room exam, patients, courts, and judges will be more sympathetic to physicians who did their best under difficult circumstances.

Front end physician involvement also makes operational sense because that's where the bottleneck is, along with the largest care gaps and the greatest treatment wins. At the front end, high-need patients receive high-benefit care. During their first few hours, ED patients are resuscitated, diagnosed, and have critical treatment initiated. Their transformation to wellness continues in the hospital, but illness severity (need) and treatment intensity (benefit) diminish. ${ }^{1}$ At the back end, where time is measured in days, stable convalescing patients consume more bed and nursing hours while accruing less health benefit (Figure 1). Whether the diagnosis is a myocardial infarction, hemothorax, or dehydration, patient need and benefit are front-loaded.

Caring for some patients while leaving others in a queue is called rationing. Ethicists believe that if rationing is necessary, priority goes to patients with the greatest need and interventions with the greatest benefit. Within this framework, need refers to a suboptimal health state and benefit to an intervention-driven outcome improvement. $^{2-4}$ The most compelling need is risk of death or disability, but acute pain also confers high priority. ${ }^{3,4}$ Thoughtful care allocation decisions might therefore prioritize, in order, lifesaving resuscitation, rapid identification of critical illness, timely pain control, definitive acute care, ongoing convalescent care, comfort, privacy, and advanced specialty treatments.

Five minutes in the ED confirms that we don't use rational frameworks to allocate care. In our system, patients no longer at risk of death or disability and those convalescing or awaiting disposition occupy the best care locations. Undiagnosed, unstabilized patients with acute pain (and often occult critical illness), who are among the sickest in the hospital when they arrive, are frequently left in hallways without care because, ostensibly, all beds are full. After they are diagnosed, treated, and stable, patients graduate to a room, a nurse, a bed, and a toilet. 


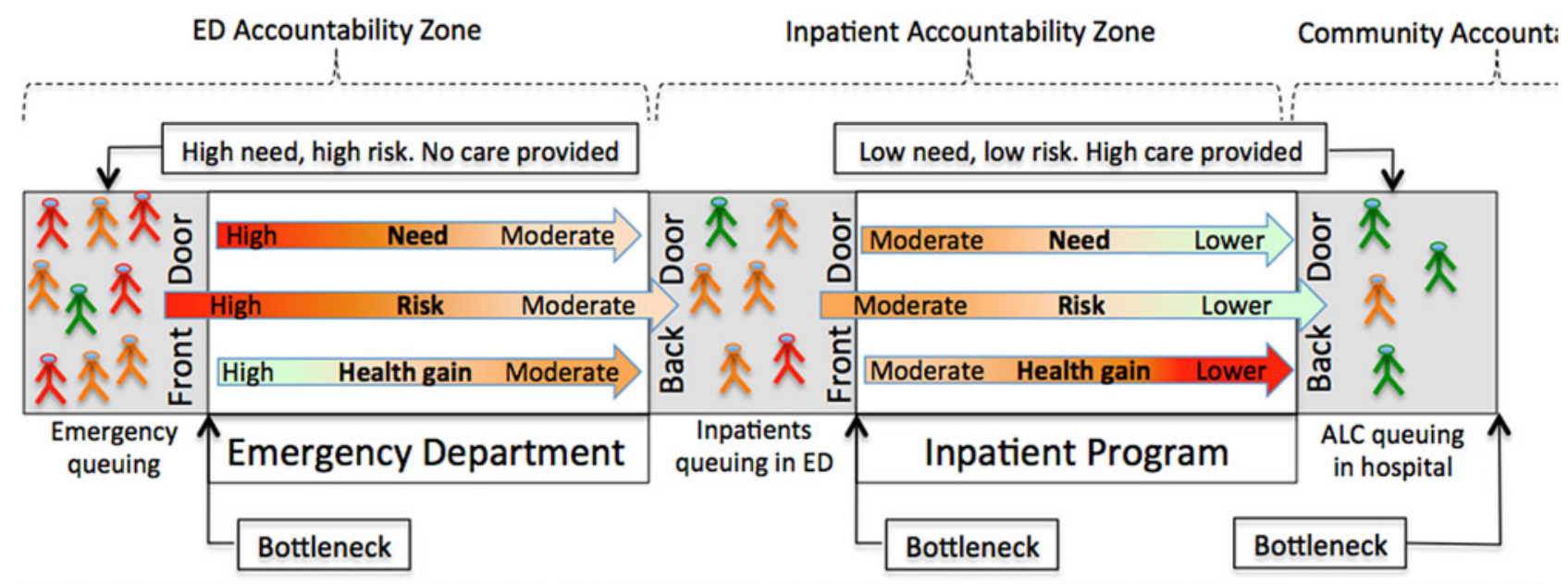

This figure illustrates patient flow through the acute care system. Risk of mortality, morbidity and severe pain (if care is not provided) are assumed to be the greatest needs. Health gain refers to improved outcomes and reduced disability resulting from care delivered. Programs have inflow and outflow points (front and back doors). Queuing patients in grey zones are the manifestation of care rationing decisions and accumulate at system bottlenecks. Adding care resources at bottlenecks improves flow and access.

Figure 1. "Needs, benefits, system constraints, and paradoxical care allocation."

No rational person would devise policies that withhold care from high-risk patients until the needs of low-risk patients (including those no longer being transformed and those awaiting placement) have been fully addressed. In a resource-constrained system, care that does not improve patient outcomes is not morally defensible, regardless of patient and provider preferences. ${ }^{4}$ Assuring comfort and privacy for convalescing patients who are accruing minimal health benefit, while simultaneously leaving suffering or acutely ill patients in waiting rooms, is a maldistribution of care that fails any ethical sniff test.

Care maldistribution occurs because we lack policies that match care provision to care needed. Such policies would incorporate at least three concepts. The first is triage, rapidly directing resources to patients with the greatest need; however, triage only works if resources are available. ${ }^{5}$ The second is reverse triage: redirecting resources from patients whose need and benefit have diminished. ${ }^{6-8}$ For example, if a stable patient is waiting for results in an ED stretcher while another is deteriorating in the hallway, this is a bad allocation decision. If a dischargeable inpatient remains in a hospital bed waiting for a test result or ride home while another patient with acute pain is suffering in a waiting room, this is a bad allocation decision. Reverse triage can free up substantial hospital resources, improve the balance of care delivery, and reduce delays for many sick patients. ${ }^{7,8}$
The third concept is adding resources at inflow bottlenecks. ${ }^{9}$ The critical resource is physicians who make diagnoses and determine dispositions. Physicians can assess patients in waiting rooms or, better, in intake zones. They can mitigate risk by recognizing serious illness that is undetectable during a triage encounter, and they can optimize stretcher allocation by identifying high-acuity patients who don't need a nurse-staffed stretcher, preserving these critical bottleneck resources for those who do. The concepts of triage, reverse triage, and attacking bottlenecks are equally relevant in the ED, hospital, and community settings.

I get it. We didn't cause this and we can't solve it. The problem is inpatients. Hospital programs should fix their capacity and efficiency shortfalls and look after their waiting patients rather than boarding them in the ED. Rising patient volumes stress the system, which should drive innovation and efficiency; however, for any program, the easy solution is to close the front door and let someone else manage the queue and make rationing decisions. ${ }^{1,10}$ I have argued to inpatient and long-term care leaders that this is a failure of program accountability, that they must develop queue management contingencies and be accountable to their waiting patients. However, if we are unwilling to do the same, to clean up our own back yard, to step outside triage and demonstrate accountability to our waiting patients, why should they? Only after you've innovated, changed processes 


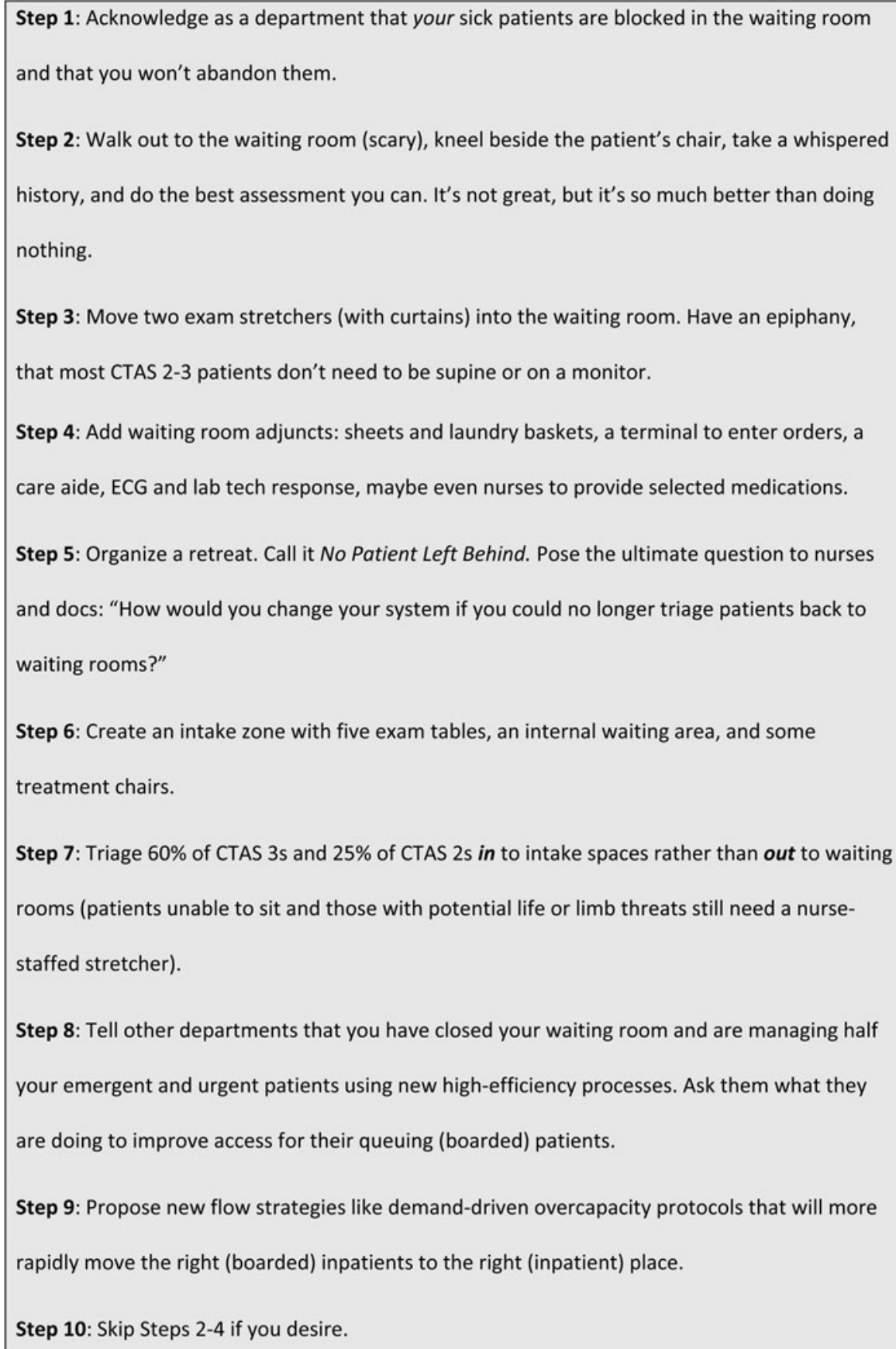

Figure 2. The evolution of waiting room care.

that weren't working any more, developed new access models, and treated 50,000 high-acuity patients in waiting rooms or intake zones, can you ask others, "What are you doing for your patients?"

Why should we go to the waiting room? To find time bombs and avert disasters. To help our triage nurses. Because it's the right thing to do. Because it's better than the alternative. Because we can. Because the greatest risk and unmet need is at the front door. Because patients appreciate it and it's what we would want for ourselves. Because it makes operational sense (Figure 2). Because physicians can make more specific risk assessments than triage nurses and preserve limited resources. Because it is leading by example, going the 
extra distance and taking responsibility for our waiting patients, demonstrating behaviours we want other programs to mimic. Because it gives us credibility and influence we wouldn't otherwise have; the currency required to push hospital-wide initiatives like overcapacity plans. Because it's the first step in changing the system.

"Don't go home yet. There are patients in your waiting room."

\section{Against: Merril Pauls (\#MerrilPauls)}

"No patient is inadequately assessed - the waiting room care doesn't address the real issues of overcrowding."

Our resources are overwhelmed on a regular basis. Our beds fill up, our waiting rooms overflow, and we fear for the safety of our patients. We know that throughput issues play a significant role in our ability to get undifferentiated patients in to assessment spaces. Everyone agrees that clearing admitted patients from our departments is the foundation of a rational, sustainable solution to ED overcrowding, and emergency medicine leaders describe a constant struggle to accomplish this.

Despite our fervent pleas, the boarding of patients continues, and we look for other solutions. For years we have practised hallway medicine - consigning assessed or admitted patients to spaces never intended for the provision of healthcare. A growing body of evidence has shown that when we put patients in hallways, closets, or repurposed offices, they receive poor care, suffer avoidable morbidity, and the level of care we provide in the department as a whole suffers. ${ }^{11-13}$ Patients and their families feel abandoned and betrayed, and suffer from the lack of privacy and dignity. ${ }^{14}$ Healthcare providers suffer moral distress as they assess and treat patients in public spaces. ${ }^{15}$ Despite all that is wrong with hallway medicine, we have normalized it. Many institutions now have protocols that acknowledge and regulate the use of hallways for patient care. ${ }^{16}$

Having lost the battle for our hallways, we turn our attention to the waiting room. We are now asking emergency physicians to venture in to the waiting room and find those sick patients that the system is failing. Consider the following narrative, which illustrates the rationale for waiting room medicine: Bold emergency doctors, distressed by long wait times and frustrated by a lack of response from hospital administration, leaves their comfortable perch in the back of the department. Their heightened diagnostic acumen allows them to find numerous critically ill patients missed by the triage process, using only a whispered history and a physical exam carried out over layers of clothing. These patients receive crucial time-sensitive interventions while the waiting room doctor moves on to identify patients who can safely be sent home. Patients are grateful because they receive the attention of a physician and believe that the indignity of a waiting room exam is a reasonable trade-off for quicker care.

We need to ask ourselves how accurate this narrative is and whether the benefits of waiting room medicine are worth the risks, as follows:

1) Are waiting room assessments good enough to find a disaster, or rule out serious pathology? When we take patients to the corner of the waiting room, or assess abdominal pain in areas with little privacy, we can't possibly be asking sensitive questions or carrying out complete exams. The argument in favor of waiting room medicine is that some care is better than none, or that we can find occult disasters in this way. But we are just as likely to be falsely reassured, and, particularly, if we are asking waiting room physicians to send people home based on these cursory assessments, we will be compromising our professional standards and compromising patient care. Physicians who have assessed patients in suboptimal conditions (in an airplane, on the side of a playing field) wonder why we can't do the same thing in our waiting rooms. Determining whether a young woman's abdominal pain may be an ectopic is different than deciding whether a football player has a dislocated shoulder. Carrying out a brief assessment and offering your best guess is appropriate for an airplane, but not an ED.

2) Is there a huge number of disasters lurking in the waiting room? Canadian EDs have been world leaders in standardizing and improving triage processes. The combination of objective measures and clinical impression that contributes to the Canadian Triage and Acuity Scale (CTAS) scoring means the vast majority of patients with clinically significant timesensitive conditions are identified early. Most EDs have adopted protocols such as electrocardiograms (ECGs) on arrival for patients with chest pain, serum beta-HCG's sent from triage for female patients, and urgent neurological consultation for patients with stroke-like symptoms. Many 
departments have reassessment teams who look for changes or deterioration in waiting room patients. Studies that look at physician-triage generally support the idea that current triage practices find the vast majority of sick patients, and physicians at the triage desk can address obvious or minor complaints promptly - but rarely find seriously ill patients who would not have been flagged otherwise. ${ }^{17,18}$

3) Is the problem finding the sick patient, or finding a place to put them? A number of months ago, I was frustrated by a lack of flow and went out to the waiting room. I identified a patient with chest pain that sounded ischemic. The ECG had been done (no ST elevation), the troponin sent, and the triage nurse knew the patient needed a monitored bed, but they were all full. She told me I could help by making another monitored bed. Our waiting rooms often overflow because there are literally no more available treatment spaces. A waiting room physician who is simply repeating the triage process or asking triage nurses to provide ongoing care in the waiting room adds little value and may interfere with other important triage processes.

Another problem is the prevalence of the conditions we are looking for, and physician practice patterns. Physicians with a higher risk-tolerance order fewer investigations and send patients home more readily. They may embrace this new role, and yet will be making riskier decisions with less information. Physicians with lower risk tolerance will find the waiting room a scary place, and may actually increase imaging and investigation rates to compensate for the cursory nature of the assessments.

4) Are patients happy trading their dignity for a quicker assessment? I suspect most patients will be grateful for the attention of a physician in the waiting room, until they find out it won't change their trajectory. If waiting rooms medicine is supposed to find the sick patient who is falling through the cracks, we will only take a small number of patients out of the queue. The vast majority will be told that they have been properly triaged and have to wait their turn - a message that many won't want to hear. I believe most patients don't just want quick care, they want accurate and definitive care. They also want care. These are hard things to provide in a brief encounter in the waiting room.

5) We shouldn't have to work in the waiting room to get others to step up, in the same way that we have normalized hallway medicine, stretchers, and curtains in the waiting room are becoming routine. When we stretch our resources and put ourselves and our patients at risk, it only seems to take the pressure off others in the system, rather than inspiring them to work harder. We are the only place in the healthcare system where there is no control over who walks through the door. Hospital administration and admitting services must accept their essential role in promoting flow through our departments, regardless of how we manage our waiting rooms.

If waiting room medicine is not the answer, how should we respond to long waits and a lack of flow? We should support improvements to current triage and waiting room processes that help us identify critically ill patients and those who need time-sensitive interventions. Physicians must make themselves available to triage nurses and charge nurses to help resolve bed block, and to discuss patients who are "falling through the cracks." There may be rare occasions where we head in to the waiting room to see a patient. But greater benefits will be realized if we work persistently and creatively in the back of our departments, to free up needed resources. Instead of drinking coffee or going home early, emergency physicians should be responding to bed block by moving the least sick patient from a monitored to non-monitored bed. We can move patients from a bed to a chair, or back to the waiting room to wait for results. We can call the computed tomography (CT) scanner, or track down the radiologist. We can call the bed coordinator or the medicine attending, or the head of surgery and ask when their patients will be moved to ward beds. Our energy and time will be better spent doing things only we can do, and letting our colleagues handle the waiting room.

Keywords: Hallway medicine, hospital crowding, triage

\section{REFERENCES}

1. Innes GD. Access block and accountability failure in the health care system. C7EM 2015;17:171-9.

2. Hasman A, Hope T, Osterdal LP. Health care need: three interpretations. F Appl Philosophy 2006;23:145-56.

3. Cookson R, Dolan P. Principles of justice in health care rationing. 7 Med Ethics 2000;26:323-9. 
4. Doyal L. Needs, rights and equity: moral quality in healthcare rationing. Qual Healthc 1995;4:273-83, doi:10. 1136/ qshc.4.4.273.

5. Lin JY, Anderson-Shaw L. Rationing of resources: ethical issues in disasters and epidemic situations. Prehosp Disaster Med 2009;24:215-21.

6. Kuschner WG, Pollard JB, Ezeje-Okoye S. Ethical triage and scarce resource allocation during public health emergencies. Hosp Topics 2007;85:16-24.

7. Kelen G, McCarthy ML, Kraus CK, et al. Creation of surge capacity by early discharge of hospitalized patients at low risk for untoward events. Disaster Med Public Health Prep 2009;3 (Suppl 1):S1-7.

8. Kelen G, Kraus CK, McCarthy ML, et al. Inpatient disposition classification for the creation of hospital surge capacity. Lancet 2006;368:1984-90.

9. Goldratt E. The goal: a process of ongoing improvement. 3rd ed. Great Barrington, MA: North River Press; 2014.

10. Innes GD. Accountability: a magic bullet for emergency care delays and healthcare access blocks. Healthc Manage Forum 2018;3:172-7.

11. Bernstein SL, Aronsky D, Duseja R, et al. The effect of emergency department crowding on clinically oriented outcomes. Acad Emerg Med 2009;16(1):1-10.
12. Sun BC, Hsia RY, Weiss RE, et al. Effect of emergency department crowding on outcomes of admitted patients. Ann Emerg Med 2013;61(6):605-11.e6.

13. Salehi L, Phalpher P, Valani R, et al. Emergency department boarding: a descriptive analysis and measurement of impact on outcomes. CFEM 2018;20(6):929-37.

14. Mah R. Emergency department overcrowding as a threat to patient dignity. CFEM 2009;11(4):365-74.

15. Anonymous. Hallway medicine. Acad Emerg Med 2008;15 (12): 1328-9.

16. Sher J. Hallway medicine becoming routine at overcrowded London hospital. The London Free Press; 2018. Available at: https://lfpress.com/news/local-news/hallway-medicine-startsin-overcrowded-london-hospitals-may-1 (accessed February 5, 2019).

17. Rowe BH, Guo X, Villa-Roel C, et al. The role of triage liaison physicians on mitigating overcrowding in emergency departments: a systematic review. Acad Emerg Med 2011;18 (2):111-20.

18. Abdulwahid MA, Booth A, Kuczawski M, Mason SM. The impact of senior doctor assessment at triage on emergency department performance measures: systematic review and meta-analysis of comparative studies. Emerg Med 7 2016;33 (7):504-13. 